\title{
Stabilization of the coupled Euler-Bernoulli plate equations with variable coefficients and time-varying delays by nonlinear internal feedback
}

Hengzhi Zhao ${ }^{1}$, Jiwei Zhang ${ }^{2}$, and Jing $\mathrm{Lu}^{1}$

${ }^{1}$ Northeast Normal University School of Mathematics and Statistics

${ }^{2}$ Yunnan University School of Mathematics and Statistics

February 28, 2022

\begin{abstract}
In this paper, we stabilize the coupled Euler-Bernoulli (E-B) plate equations with variable coefficients and time-varying delays by defining a suitable energy function based on Riemannian geometry.
\end{abstract}

\section{Hosted file}

New_Manucript_LaTeX.pdf available at https://authorea.com/users/462709/articles/558068stabilization-of-the-coupled-euler-bernoulli-plate-equations-with-variable-coefficientsand-time-varying-delays-by-nonlinear-internal-feedback 